\title{
Correlated spectral and temporal changes in 3C 390.3: a new link between AGN and Galactic black hole binaries?
}

\author{
M. Gliozzi ${ }^{1}$, I. E. Papadakis ${ }^{2}$, and C. Räth ${ }^{3}$ \\ ${ }^{1}$ George Mason University, Department of Physics \& Astronomy \& School of Computational Sciences, 4400 University Drive, MS 3F3, \\ Fairfax, VA 22030, USA \\ e-mail: mario@physics.gmu.edu \\ 2 Physics Department, University of Crete, 71003 Heraklion, Crete, Greece \\ 3 Max-Planck-Institut für extraterrestrische Physik, Postfach 1312, 85741 Garching, Germany
}

Received 17 May 2004 / Accepted 22 November 2005

\section{ABSTRACT}

We present the results of a systematic search for evidence of temporal changes (i.e., non-stationarity) associated with spectral variations in 3C 390.3, using data from a two-year intensive RXTE monitoring campaign of this broad-line radio galaxy. In order to exploit the potential information contained in a time series more efficiently, we adopt a multi-technique approach, making use of linear and non-linear techniques. All the methods show suggestive evidence of non-stationarity in the temporal properties of 3C 390.3 between 1999 and 2000 , where the characteristic time-scale of variability decreases as the energy spectrum of the source softens. However, only the non-linear, "scaling index method" is able to conclusively show that the temporal characteristics of the source do vary, although the physical interpretation of this result is not clear at the moment. Our results indicate that the variability properties of 3C 390.3 may vary with time, in the same way as they do in Galactic black holes in the hard state, strengthening the analogy between the X-ray variability properties of the two types of object. This is the first time that such a behavior is detected in an AGN X-ray light curve. Further work is needed to investigate whether this is a common behavior in AGN, as it is in the Galactic binaries.

Key words. galaxies: active - galaxies: nuclei - X-rays: galaxies

\section{Introduction}

Among the basic properties characterizing an Active Galactic Nucleus (AGN) the flux variability, especially at X-rays, is one of the most commonly used. Previous multi-wavelength variability studies (e.g., Edelson et al. 1996; Nandra et al. 1998) have shown that AGN are variable in every observable wave band, but the X-ray flux exhibits variability of larger amplitude and on time-scales shorter than any other energy band, indicating that the X-ray emission originates in the innermost regions of the central engine.

However, even though the discovery of X-ray variability dates back more than two decades, its origin is still poorly understood. Nevertheless, it is widely acknowledged that the variability properties of AGN are an important means of probing the physical conditions in their emission regions. The reason is that using energy spectra alone it is often impossible to discriminate between competing physical models and thus the complementary information obtained from the temporal analysis is crucial to break the spectral degeneracy.

Over the years, several variability models have been proposed, involving one or a combination of the fundamental components of an AGN: accretion disk, corona, and relativistic jet.
These models can be divided into two main categories: 1) intrinsically linear models, such as the shot noise model (e.g., Terrel 1972), where the light curve is the result of the superposition of similar shots or flares produced by many independent active regions; and 2) non-linear models, which require some coupling between emitting regions triggering avalanche effects, such as the self-organized criticality model (e.g., Mineshige et al. 1994), or models assuming that the variability is caused by variations in the accretion rate propagating inwards (e.g. Lyubarskii 1997). The "rms-flux relation" recently discovered by Uttley \& McHardy (2001), along with detection of nonlinearity in different AGN light curves (i.e., in 3C 345 by Vio et al. 1992; in 3C 390.3 by Leighly \& O'Brien 1997; in NGC 4051 by Green et al. 1999; in Ark 564 by Gliozzi et al. 2002), favors non-linear variability models, but there is no general consensus on the nature of the X-ray variability yet.

Because of their brightness, the temporal and spectral properties of Galactic black holes (GBHs) are much better known and can be used to infer information on their more powerful, extragalactic analogs, the AGN. It is well established that GBHs undergo state transitions (see McClintock \& Remillard 2003 for a recent review), switching between "low", "intermediate", "high" and "very high" states, which are all 
unambiguously characterized by a specific combination of energy spectrum and power spectral density (PSD). Importantly, even when in the same state, the timing properties of GBHs may vary with time, often accompanying changes of the spectral properties. For example, Pottschmidt et al. (2003) showed that changes in the PSD of Cyg X-1 are associated with changes in the slope of the energy spectrum. Specifically, during the "low/hard state", the PSD characteristic frequencies increase, as the spectral slope becomes steeper.

Transitions between "different" states have not been observed yet in AGN. The reason may be associated with the fact that their flux variability is characterized by much longer timescales. Nevertheless, a correspondence between GBH spectral states and some AGN classes has been hypothesized. Specifically, Broad-Line Seyfert 1 galaxies have been associated with the GBH low/hard state, and Narrow-Line Seyfert 1 galaxies with the GBH high/soft state (for a recent discussion on this topic see, for example, McHardy et al. 2004).

Although genuine spectral transitions in AGN are unlikely to be detected due to the longer time-scales involved, it is perhaps possible to detect changes in the temporal properties (i.e., loss of stationarity) associated with spectral changes, similarly to what is observed in GBHs within the same spectral state. To this end, the numerous monitoring campaigns carried out by $R X T E$ in the last decade are crucial, since they have produced data sets suitable for searching for non-stationarity in the AGN light curves, where long-term spectral changes are nearly ubiquitous.

In this work, we present the results from a systematic search for evidence of temporal changes associated with significant spectral variations in the case of the broad-line radio galaxy (BLRG) 3C 390.3. We use data from a two-year intensive $R X T E$ monitoring campaign. Previous X-ray studies with ROSAT and RXTE (Leighly \& O'Brien 1997; Gliozzi et al. 2003) demonstrated that 3C 390.3 is one of the most variable AGN on time-scales of days and months. Our motivation stems from the results of a long monitoring campaign carried out with ROSAT HRI, which showed evidence for possible nonstationarity (and non-linearity) in the soft X-ray light curve of 3C 390.3 (Leighly \& O’Brien 1997).

\section{Observations and data reduction}

We use archival RXTE data (PI: Leighly) of 3C 390.3. This source was observed by $R X T E$ for two consecutive monitoring campaigns between 1999 and 2001. The first set of observations was carried out from 1999 January 8 to 2000 February 29, and the second one from 2000 March 3 to 2001 February 23. Both campaigns were performed with similar sampling: 3C 390.3 was regularly observed for $\sim 1000-2000$ s once every three days. The observations were carried out with the Proportional Counter Array (PCA; Jahoda et al. 1996) and the High-Energy X-Ray Timing Experiment (HEXTE; Rotschild et al. 1998) on RXTE. Here we will consider only PCA data, because the signal-to-noise of the HEXTE data is too low for a meaningful timing analysis.

The PCA data were screened according to the following acceptance criteria: the satellite was out of the South
Atlantic Anomaly (SAA) for at least $30 \mathrm{~min}$, the Earth elevation angle was $\geq 10^{\circ}$, the offset from the nominal optical position was $\leq 0^{\circ} 02$, and the parameter ELECTRON-2 was $\leq 0.1$. The last criterion removes data with high particle background rates in the Proportional Counter Units (PCUs). The PCA background spectra and light curves were determined using the L7-240 model developed at the RXTE Guest Observer Facility (GOF) and implemented by the program pcabackest v.2.1b. Since the two monitoring campaigns span three different gain epochs, the appropriate files (pca_bkgd_cmfaint17_e3 $(4,5)$ bv20031123.mdl, depending on the observation date), provided by the RXTE Guest Observer Facility (GOF), were used to calculate the background light curves. This model is appropriate for "faint" sources, i.e., those producing count rates less than $40 \mathrm{~s}^{-1} \mathrm{PCU}^{-1}$. All the above tasks were carried out using the FTOOLS v.5.1 software package and with the help of the rex script provided by the RXTE GOF, which also produces response matrices and effective area curves appropriate for the time of the observation. Data were initially extracted with $16 \mathrm{~s}$ time resolution and subsequently re-binned at different bin widths depending on the application. The current temporal analysis is restricted to PCA, STANDARD-2 mode, 2-20 keV, Layer 1 data, because that is where the PCA is best calibrated and most sensitive. Since PCUs 1, 3, and 4 were frequently turned off (the average number of PCUs is 2.25 and 1.95 in the 1999 and 2000 observations, respectively), only data from the other two PCUs (0 and 2 ) were used. All quoted count rates are for two PCUs.

The spectral analysis of PCA data was performed using the XSPEC v. 11 software package (Arnaud 1996). We used PCA response matrices and effective area curves created specifically for the individual observations by the program pcarsp, taking into account the evolution of the detector properties. All the spectra were re-binned so that each bin contained enough counts for the $\chi^{2}$ statistic to be valid. Fits were performed in the energy range 4-20 keV, where the signal-to-noise ratio is the highest.

\section{The X-ray light curve}

Figure 1 shows the background-subtracted $2-20 \mathrm{keV}$ count rate and hardness ratio $(\mathrm{HR}=7-20 \mathrm{keV} / 2-5 \mathrm{keV})$ light curves of 3C 390.3. Filled circles represent data points from the first monitoring campaign; open diamonds are used for the second campaign. A visual inspection of the HR and count rate light curves indicates the presence of a typical Seyfert-like spectral evolution (the energy spectrum softens when the source brightens), and suggests the existence of different variability patterns during the two monitoring campaigns. In the first one, 3C 390.3 exhibits an initial smooth decrease in the count rate (a factor $\sim 2$ in 100 days) followed by an almost steady increase (a factor $\sim 3.5$ in 100 days). After day 200 (from the beginning of the observation), the source shows significant, low-amplitude, fast variations. During the second monitoring campaign, the source does not show any long-term, smooth change in count rate, but only a flickering behavior with two prominent flares. This qualitative difference is associated with 


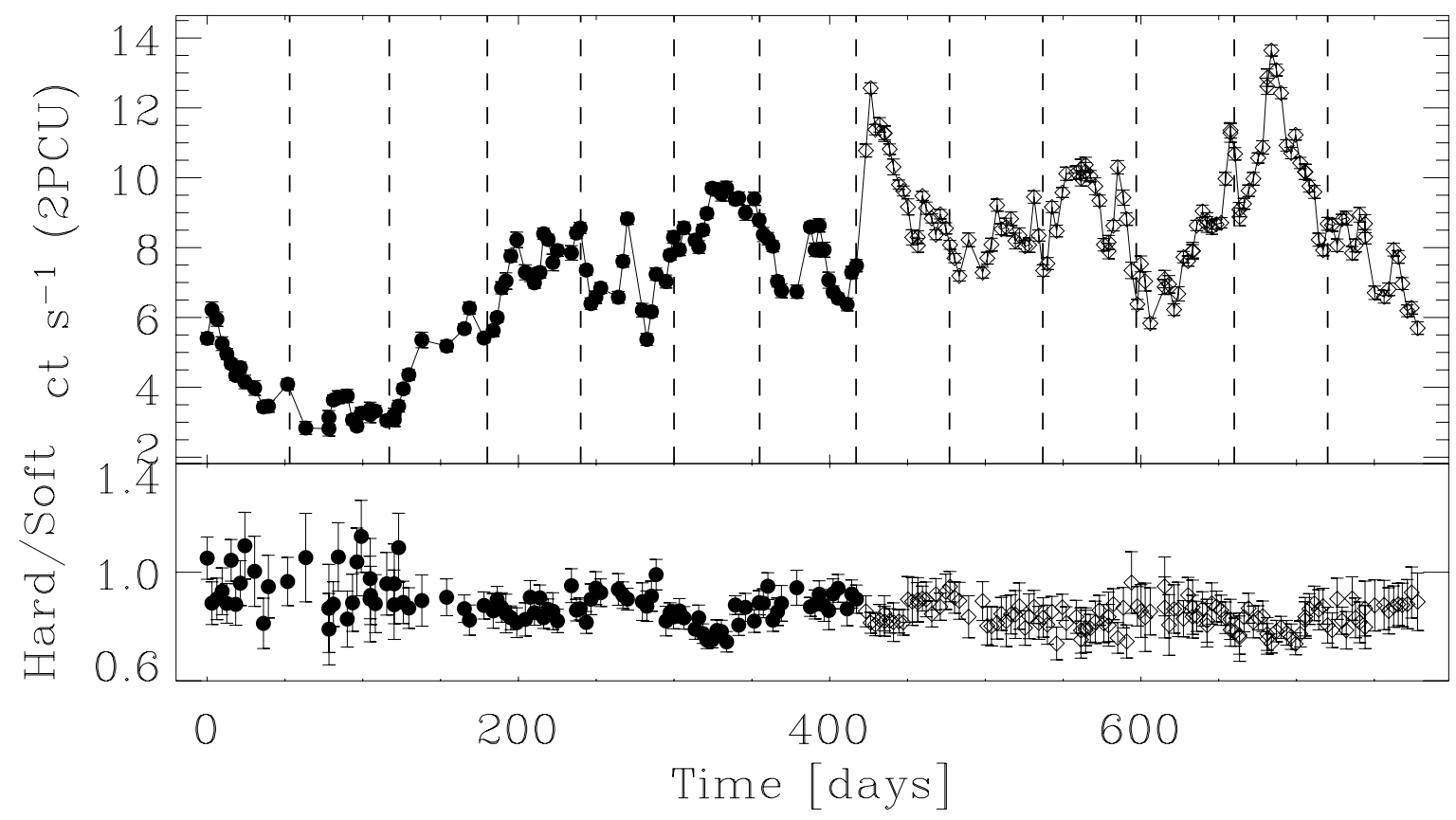

Fig. 1. X-ray light curves of the background-subtracted count rate in the $2-20 \mathrm{keV}$ band (top panel) and of the $\mathrm{X}$-ray color 7-20 keV/2-5 keV (bottom panel) from RXTE PCA observations of 3C 390.3. Time bins are $5760 \mathrm{~s}$ ( 1 RXTE orbit). Filled circles represent data points from the first monitoring campaign from 1999 January 8 to 2000 February 29, whereas open diamonds are data points from the second monitoring campaign from 2000 March 3 to 2001 February 23. The dashed lines in the top panel indicate the intervals used for the time-resolved spectral analysis (see Sect. 9).

marked differences in the two mean count rates, $6.4 \pm 0.2 \mathrm{~s}^{-1}$ and $9.0 \pm 0.1 \mathrm{~s}^{-1}$, and in the fractional variability amplitudes, $F_{\text {var,99 }}=(33 \pm 3) \%$ and $F_{\text {var,00 }}=(19 \pm 2) \%$, calculated over each campaign (see, e.g., Vaughan et al. 2003 for a detailed description of $F_{\text {var }}$ and the estimate of its uncertainty). The uncertainties have been computed assuming that the data distribution is normal and the data are a collection of independent measurements. However, the data points of AGN light curves are not independent but correlated and thus the apparent difference between the mean count rates and $F_{\text {var }}$ could simply result from the red-noise nature of the variability process, combined with its randomness (see, e.g., Vaughan et al. 2003).

\section{Stationarity tests}

Almost all methods employed in timing analysis (e.g., power spectrum, structure function, auto-correlation analysis) assume some kind of stationarity. Generally speaking, a system is considered stationary if the average statistical properties of the time series are constant with time. More specifically, the stationarity is defined as "strong" if all the moments of the probability distribution function are constant, and "weak" if only the moments up to the second order (i.e., mean and variance) are constant. Usually, non-stationarity is considered an undesired complication. However, in some cases the non-stationarity provides the most interesting information, as in the case of GBHs switching from one spectral state to another.

In previous temporal studies of AGN, no strong evidence for non-stationarity has been reported. Indeed, AGN light curves are considered to be "second-order" stationary, due to their red-noise variability. Here, we adopt several complementary methods to investigate the issue of the stationarity in the 3C 390.3 light curve. First, the temporal properties are studied with traditional linear techniques in the Fourier domain, by estimating the PSD, and in the time domain with the structure function analysis. Secondly, we directly probe the probability density function associated with the time series. Finally, we utilize the scaling index method, a technique borrowed from non-linear dynamics, useful for detecting weak signals in noisy data.

All the above methods are separately applied to the 1999 and 2000 monitoring campaigns. We quantify the differences between the estimated functions (like the power spectrum, the structure function, etc.) using appropriate "statistics". The probability that these statistics will have the observed values are rigorously estimated with extensive Monte Carlo simulations. These simulations allow us to assess whether the observed values are due to measurement uncertainties (i.e., Poisson noise), to the light curve sampling, to the red-noise nature of the variability process, or to intrinsic variations of the statistical properties of the source.

\subsection{Analysis in the Fourier domain: power spectral density}

We followed the method of Papadakis \& Lawrence (1993) to compute the power spectrum of the 1999 and 2000 light curves, after normalizing them to their mean. We used a 3-day binning (i.e., equal to the typical sampling rate of the source) for both light curves. As a result, the light curves that we used are evenly sampled, but there are a few missing points in them; 30 out 


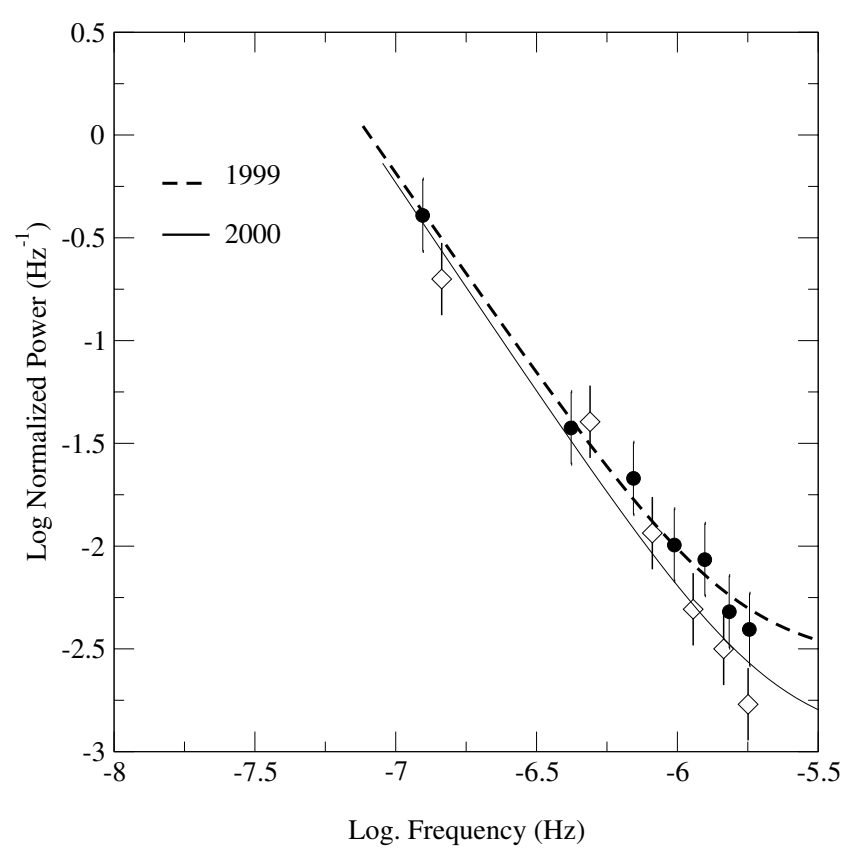

Fig. 2. RXTE PCA power density spectra using based on the 1999 and 2000 light curves with 3-day bins, normalized to their mean (filled circles and open diamonds, respectively). The solid and dashed lines show the best fitting power law models, including the effects of the experimental Poisson noise level.

of 140 and 15 out of 120 for the 1999 and 2000 light curves, respectively. These points are randomly distributed over the whole light curve, and we accounted for them using a linear interpolation between the two bins adjacent to the gaps, adding appropriate Poisson noise.

Figure 2 shows the PSD of the 1999 and 2000 light curves (filled circles and open diamonds, respectively). Each point in the estimated PSD represents the average of 10 logarithmic periodogram estimates. We fitted both spectra with a simple power law $\left(P(f) \propto f^{-a}\right)$. The model provides a good description of both PSDs; the best fitting slope values (shown in Fig. 2) are consistent within the errors with the value of $2 \pm 0.3$ in both cases.

A closer look at the PSD plot indicates a possible low frequency flattening of the 2000 PSD between $\log (v)=$ -6.5 and -7 , corresponding to a time interval between $\sim 35$ and 115 days. In order to investigate this issue further we split the two lowest frequency points into two (to increase the frequency resolution) and refitted the 2000 PSD with a broken power-law model. We kept the slope below and above the frequency break fixed to the values 1 and 2, respectively, and kept as free parameters the break frequency and normalization of the PSD. The model provides a good fit to the 2000 PSD, but statistically not better than a simple power law according to an F-test. The best fitting break frequency is $2.5 \times 10^{-7} \mathrm{~Hz}$, which corresponds to a break time-scale of $\sim 46$ days. The $90 \%$-confidence lower limit to this time-scale is 33 days, while, due to the limited coverage of the observed PSD at lower frequencies, the upper limit is undefined. We also tried to fit the 1999 PSD with this model, but without success: the best fitting break frequency turned out to be lower than the lowest frequency sampled by the 1999 light curve, indicating that there is no indication of any breaks in the 1999 PSD. This power spectrum follows a power-law shape with slope $a=2$ down to the lowest sampled frequencies.

In order to quantify the comparison between the 1999 and 2000 PSDs in a statistical way, we used the " $S$ " statistic as defined by Papadakis \& Lawrence (1995). To avoid the constant Poisson noise level, we considered the periodogram estimates up to frequencies $\sim 7 \times 10^{-7} \mathrm{~Hz}$. We find that $S=-1.3$, which implies that the two light curves are not far away from the hypothesis of stationarity. Therefore, the differences suggested in the previous paragraph by the broken power-law model fitting results are not supported by this more rigorous statistical test. Light curves with denser sampling, which would result in a much higher frequency resolution at lower frequencies (where the potential differences between the two PSDs are observed), would be necessary for a more sensitive comparison between the 1999 and 2000 power spectra.

Recent results from the power spectrum analysis of combined XMM-Newton and RXTE light curves of radio-quiet AGN (Markowitz et al. 2003; McHardy et al. 2004) show that the $2-10 \mathrm{keV}$ power spectrum of AGN follows a power-law form with a slope of 2 down to a "break" frequency, below which it flattens to a slope of 1 . This break time-scale appears to scale with the black hole mass according to the relation: $t_{\mathrm{br}}$ (days) $=M_{\mathrm{BH}} / 10^{6.5} M_{\odot}$ (Markowitz et al. 2003). Using the reverberation mapping technique, Peterson et al. (2004) estimate the black hole mass of 3C 390.3 to be $M_{\mathrm{BH}} \sim$ $2.9 \times 10^{8} M_{\odot}$. In this case, the characteristic break time-scale of 3C 390.3 should be $\sim 90$ days, which corresponds to a break frequency of $\sim 10^{-7} \mathrm{~Hz}$. This break frequency is entirely consistent with the broken power-law model fitting results in the case of the 2000 light curve. Unfortunately, though, even the combined 1999 and 2000 PSD does not extend to frequencies low enough for this break frequency to be firmly detected.

The value assumed for the mass of the black hole harbored by 3C 390.3 is still a matter of debate. The value used in this work is the most recent and it is consistent within the errors with the mass estimate given by Kaspi et al. (2000). However, based on another reverberation mapping experiment on this source, Sergeev et al. (2002) find a longer reverberation lag and determine a higher mass of $2 \times 10^{9} M_{\odot}$ using a different method. In that case, the putative break frequency in the PSD would be located at a frequency of $\sim 1 / \mathrm{yr}$, which could not be probed with the current data set.

We conclude that the combined $1999+2000,2-20 \mathrm{keV}$ PSD of 3C 390.3 is well fitted by a simple power-law model at all sampled time-scales from $3^{-1}-100^{-1}$ days $^{-1}$. The same model fits well the individual 1999 and 2000 PSDs as well. There is an indication of a low-frequency PSD break (at a frequency which corresponds to a time-scale of $\sim 50$ days) in the 2000 PSD, which is absent in the 1999 PSD. However, due to the low resolution of the power spectra at hand, we cannot be certain of the significance of the difference between the two PSDs. 


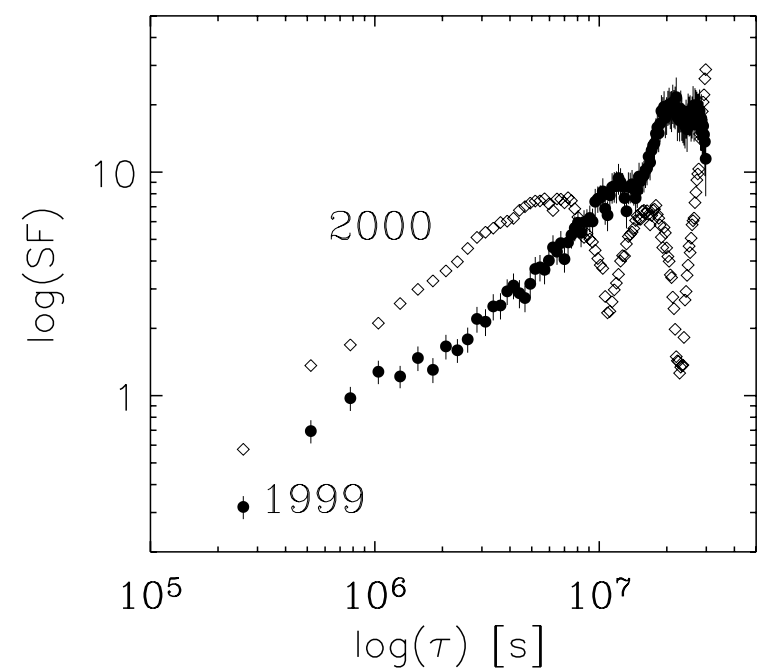

Fig. 3. Structure function of 3C 390.3 during the 1999 (filled circles) and 2000 (open diamonds) monitoring campaign. Time bins are 3 days. For clarity, error-bars were plotted only for the 1999 SF.

\subsection{Analysis in the time domain: structure function}

Next, we performed an analysis based on the structure function (e.g., Simonetti 1985), a linear method that works in the time domain and has the ability to discern the range of timescales that contribute to the variations in the data set. In principle, the SF should provide the same information as the PSD, as both functions are related to the auto-covariance function of the process $R(\tau)$. The power spectrum is defined as the Fourier transform of $R(\tau)$, while the intrinsic $\mathrm{SF}$ at lag $\tau$ is equal to $2 \sigma^{2}-R(\tau)$, where $\sigma^{2}$ is the variance of the process (Simonetti et al. 1985). In fact, if the PSD follows a power law of the form $P(f) \propto f^{-a}$ (where $f$ is the frequency), then $\mathrm{SF} \propto \tau^{a-1}$ (Bregman et al. 1990).

We computed the structure functions for the first and second monitoring campaigns. The results are plotted in Fig. 3: the filled circles refer to the 1999 light curve, whereas the open diamonds describe the 2000 SF. Both SFs have a power-law shape of the form $\mathrm{SF} \propto \tau^{1}$, which is consistent with the results of the PSD analysis, since $P(f) \propto f^{-2}$ (see Sect. 4.1). Furthermore, the $2000 \mathrm{SF}$ shows a flattening to $\mathrm{SF} \propto \tau^{0}$, at a characteristic time lag of $\sim 4-6 \times 10^{6} \mathrm{~s}$, followed by an oscillating behavior. This value corresponds to a characteristic time-scale of $\sim 50-70$ days, which is fully consistent with the putative break suggested by the 2000 PSD and corresponds approximately to the value expected on the basis of the black hole mass estimate (see discussion in Sect. 3.1).

The 1999 and 2000 SFs look qualitatively different. However, the statistical significance of this difference cannot be assessed directly, since the the SF points are correlated and their true uncertainty is unknown. The errors shown in Fig. 3 are representative of the typical spread of the points around their mean in each time-lag bin, hence they depend mainly on the the number of points that contribute to the SFs estimation at each time lag. To investigate in a quantitative way whether the difference between the two SFs can be simply ascribed to the intrinsic, red-noise randomness of the light curves or if it actually reveals a non-stationary behavior, the use of Monte Carlo simulations is necessary.

For this reason, we performed a model-dependent numerical experiment assuming that both the 1999 and 2000 light curves are realizations of a process with mean count rate equal to the average value of the 1999 and 2000 light curves, and with a power spectrum of power-law index 2 down to a break frequency below which the power-law index changes to 1 . We performed the experiment considering various PSD break time scales: $t_{\mathrm{br}}=50,100,200$, and $300 \mathrm{~d}$. Here we present only the results from $t_{\mathrm{br}}=300 \mathrm{~d}$ because they provide the most conservative limits on the significance of the observed differences. In all cases, the PSD normalization was fixed in such a way that the model PSD will have the same value as the observed $1999+2000$ best fitting power law at $10^{-6} \mathrm{~Hz}$. Assuming this power spectrum model, we created two sets of $10^{3}$ synthetic light curves (using the method of Timmer \& Koenig 1995). Importantly, for each synthetic light curve, the appropriate RXTE background light curve (as provided by the FTOOL PCABACKEST) was added to each light curve, and then the appropriate Poisson noise was added to each point in them. The length of the synthetic light curves was 20 times longer than the length of the observed light curves, and their points were separated by intervals of $160 \mathrm{~s}$. In this way, we could simulate the effects of the red noise leakage in the estimation of the SF, although the aliasing effects were not accounted for. This should not be a serious problem though, as the steep power spectral slope (-2) implies that these effects should not be very strong. Each synthetic light curve was then re-sampled so as to have the same length and sampling pattern as the observed light curves.

After fixing the PSD normalization, the synthetic light curves are not renormalized to the observed variance, but their variance is free to vary, allowing a larger scatter in the lowfrequency power and hence maximizing the effects of longterm flux variations. For completeness, we also performed a second set of "standard" red-noise simulations with synthetic light curves renormalized so as to have the same sampling pattern, mean and variance as the 1999 and 2000 light curve, respectively. No significant differences in the red-noise tests of SF are found using the two different numerical approaches.

For each pair of synthetic light curves, we computed their SFs and used the sum of the squared ratios between them at each lag, i.e. $D_{\mathrm{SF}}=\sum\left[\mathrm{SF}_{2}\left(\tau_{\mathrm{i}}\right) / \mathrm{SF}_{1}\left(\tau_{\mathrm{i}}\right)\right]^{2}$, to quantify their difference. Based on the distribution of the $D_{\mathrm{SF} \text {,synth values (see }}$ Fig. 4), we find that the probability that the $D_{\mathrm{SF}, \text { obs }}$ value would be as large as observed, is of the order of $44 \%$. Therefore, the apparent differences between the observed 1999 and 2000 SFs shown in Fig. 3 can be the result of the red-noise character of the variability process.

However, we noticed that the synthetic SFs show the largest differences at lags larger than $\sim 100$ days (i.e., at those lags where the wiggles and bumps in the observed $\mathrm{SF}_{00}$ appear). When we restricted the computation of the $D_{\mathrm{SF}}$ up to lag = 90 days (thus including the flattening of the observed $S F_{00}$, but excluding the part that shows the highly erratic behavior at large lags) we found that probability of the difference between the observed 1999 and 2000 SFs being due to red-noise effect decreases to $7.5 \%$. 


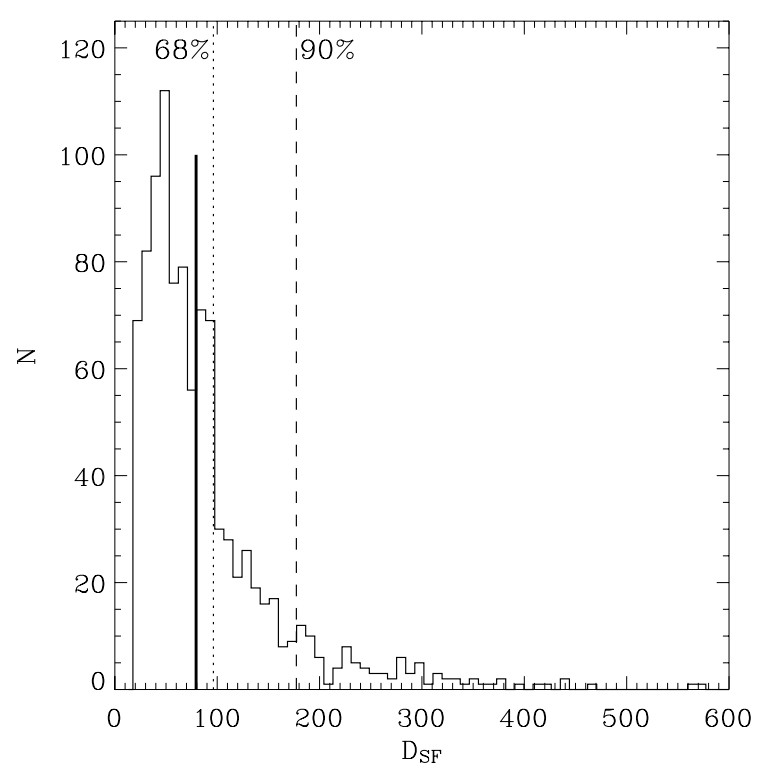

Fig. 4. Comparison between $D_{\mathrm{SF}, \text { obs }}$ (thick continuous line) and the distribution of $D_{\mathrm{SF}, \text { synth }}$ obtained with red-noise simulations.

The SF analysis results are similar to those from the power spectrum analysis. The $\mathrm{SF}_{00}$ shows a flattening around lag 50-70 days, which is absent in the $\mathrm{SF}_{99}$, suggesting that the power spectrum break may have indeed moved to higher frequencies during the 2000 observations, in agreement with the 1999 and 2000 PSDs. However, as with the observed PSDs, we cannot be certain of the reality of this effect.

\subsection{Time-independent analysis: probability density function}

After utilizing methods based on the temporal order of the data points, here we investigate the stationarity of 3C 390.3 with a method independent of the temporal order of the data points, the probability density function (PDF), which is the histogram of the different count rates recorded during the monitoring campaign. Figure 5 shows the normalized distributions $(r-\langle r\rangle) /\langle r\rangle$ of the 1999 (thick solid line) and 2000 (dotted line) monitoring campaigns. The first transformation $(r-\langle r\rangle)$ brings both light curves to zero mean, the second, normalizes the amplitudes to unit mean. In this way, the relative amplitudes of the light curves are directly probed, and the PDF results can be directly compared to the power spectral ones, which use exactly the same convention.

For a better visual comparison, we have used small time bins of $32 \mathrm{~s}$ in Fig. 5. However, for a quantitative comparison between PDFs (see below), we have used larger time bins to prevent any contamination from the Poisson noise. There is a clear qualitative difference between the two PDFs, with the 1999 distribution characterized by two broad peaks and the 2000 PDF having a bell-like shape. This qualitative difference is confirmed by a Kolmogorov-Smirnov (K-S) test, which gives a probability of $99.99 \%$ that the two distributions are different.

One may ask what the effects on the PDF properties of the light curve bin size are, whether the PDF is really time

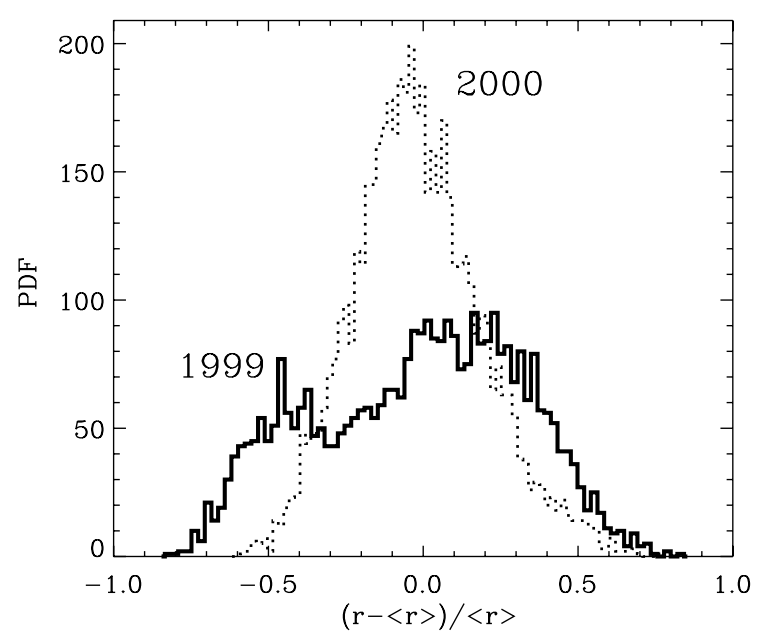

Fig. 5. Probability density function of count rates scaled and normalized with respect to the average count rate $\langle r\rangle$, during the 1999 (thick solid line) and 2000 (dotted line) monitoring campaign. The time bins are $32 \mathrm{~s}$ wide.

independent (a question that is directly related to the length of the light curve), and whether the K-S test is appropriate to quantify the statistical difference between two PDFs or not. The answers to these questions are not clear. In all cases, the most important issue is the red-noise character of the AGN light curves (in all wavebands). As the size of the light curve bins decreases, the number of the points in the PDF increases, and the sampled PDF is better defined. However, as the points added are heavily correlated due to the red-noise character of the variations, no "extra" information is added to the PDF. On the other hand, since the "sensitivity" of the K-S test increases with the number of points in the sampled PDF (which are assumed to be independent), there is a possibility that differences that are purely due to the stochastic nature of a possible stationary process will be magnified and the K-S test will erroneously indicate "significant" differences between two PDFs. Finally, one should also consider the effects of the Poisson noise on the sampled PDFs. In the cases where the source signal has a much larger amplitude than the variations caused by the experimental noise (as is the case here), we should not expect this effect to be of any significance. The Poisson noise should produce similar PDFs (once they are normalized to their mean) so any significant differences cannot be due to this effect.

To assess the apparent PDF differences shown in Fig. 5 in a more rigorous way, we have used the $10^{3}$ synthetic light curves with tbin $=160 \mathrm{~s}$, obtained from the Monte Carlo simulations described in Sect. 4.2. For each pair of synthetic PDFs, we computed the sum $D_{\mathrm{PDF}}=\sum\left[\mathrm{PDF}_{1}(i)-\mathrm{PDF}_{2}(i)\right]^{2}$, in order to quantify their differences. A comparison of the distribution of the $10^{3}$ synthetic $D_{\mathrm{PDF} \text {,synth }}$ with the observed value $D_{\mathrm{PDF}, \text { obs }}$ (represented by a thick solid line in Fig. 6) reveals that a value as large as $D_{\mathrm{PDF}, \text { obs }}$ appears in $14.3 \%$ of all the synthetic light curves. Consequently, the apparent difference between $\mathrm{PDF}_{99}$ and $\mathrm{PDF}_{00}$ could be caused by red-noise effects.

Looking closer at the synthetic PDFs that show the largest $D_{\mathrm{PDF}}$ values, we noticed that they are caused mainly by differences at $|(r-\langle r\rangle) /\langle r\rangle|>0 / 6$, where there is only a small 


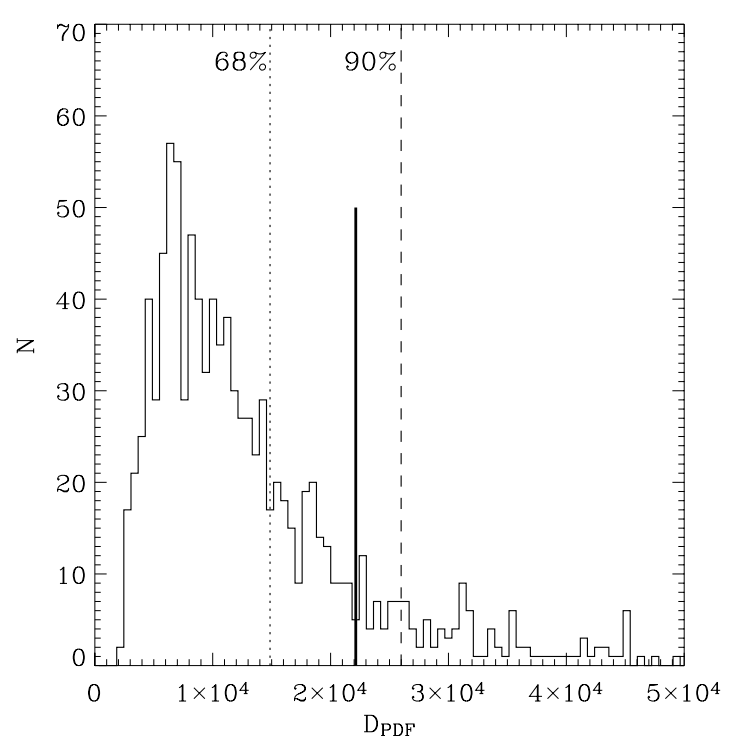

Fig. 6. Comparison between $D_{\mathrm{PDF}, \text { obs }}$ (thick continuous line) and the distribution of $D_{\mathrm{PDF}, \text { synth }}$ obtained with red-noise simulations.

fraction of the total number of points in the histograms. For that reason, we divided the $x$-axis into three sub-intervals $(-1 /-0.6$; $-0.6 / 0.6$; and $0.6 / 1$ ) and calculated $D_{\mathrm{PDF}}$ in each one of them. When we consider the middle interval, the probability that the difference between the observed PDFs can be explained to rednoise effects is reduced to $12.6 \%$.

In summary, the 1999 PDF appears to be broader than the 2000 PDF, which shows a well defined, uniform distribution around its mean. It shows a bimodal shape, with a large fraction of points having a count rate smaller than average. This is qualitatively consistent with the results from the previous sections. For example, in the case of a PSD break at low frequencies, the observed variations are dominated by the components with long periods. If the PDF is estimated with data sets shorter than the PSD break time-scale, we would expect the points to show a broad distribution around the light curve mean, as in the case of the 1999 PDF. Indeed, our results in the case of a PSD break at $1 / 50$ days $^{-1}$ show that the observed 1999 PDF is entirely inconsistent with this assumption, contrary to the observed 2000 PDF which is consistent. However, as before with the results from the PSD and SF analysis, a red-noise process with a PSD break at $1 / 300$ days $^{-1}$ is in rough agreement with both the 1999 and 2000 PDFs. So, we conclude that observed 1999 and 2000 PDF differences possibly support the hypothesis of non-stationarity, but not at a high significance level.

\subsection{Non-linear analysis: scaling index method}

Non-linear methods are rarely employed in the analysis of AGN light curves, partly because these methods are less developed than linear ones, partly because it is implicitly assumed that AGN light curves are linear and stochastic. However, nonlinear methods can be useful not only to characterize the nature of chaotic deterministic systems, but also to discriminate between two time series, regardless if they are linear or non-linear.
When methods of non-linear dynamics are applied to time series analysis, the concept of phase space reconstruction represents the basis for most of the analysis tools. To study chaotic deterministic systems it is important to establish a vector space (the phase space) such that specifying a point in this space specifies a state of the system, and vice versa. However, in order to apply the concept of phase space to a time series, which is a scalar sequence of measurements, one has to convert it into a set of state vectors.

For example, let us suppose we have an observed time series, $x\left(t_{i}\right)$, with $i=\Delta t, 2 \Delta t, \ldots, N \Delta t$. One can construct a set of $2(3,4, \ldots)$-dimensional vectors by selecting pairs (triplets, quadruplets, ...) of data points whose count rate values represent the values of the different components of each vector. The data points defining the vector components are chosen such that the second point is separated from the first by a time delay $\Delta t^{\prime}$, while the third is separated from the first by $2 \Delta t^{\prime}$, and so on. This process, called time-delay reconstruction, can be easily generalized to any $n$-dimensional vector. The time delay $\Delta t^{\prime}$ is usually much larger than the sampling interval $\Delta t$ of the observed light curve. In non-linear dynamics, where this technique is used to determine fractal dimensions and discriminate chaos from stochasticity, the choice of the time delay is not arbitrary, but should satisfy specific rules (see Kantz \& Schreiber 1997, for a review).

The set of $n$-dimensional vectors, derived from the original light curve, is then mapped (or embedded) into an $n$-dimensional vector space, producing a phase space portrait whose topological properties represent the temporal properties of the corresponding time series.

A widely used method to quantify the characteristics of a phase space portrait is the correlation integral $C(R)$ (Grassberger \& Procaccia 1983). An exhaustive description of the correlation integral method and its application to X-ray light curves of AGN is given by Lehto et al. (1993).

Another method that can quantify the topological properties of a phase space portrait is the scaling index method (hereafter SIM; e.g., Atmanspacher et al. 1989) which has been employed in a number of different fields (e.g., Räth \& Morfill 1997; Räth et al. 2002), including the study of AGN time series in the case of Ark 564 (Gliozzi et al. 2002). Unlike the correlation integral, which is a global indicator, the scaling index method measures both the global and local properties of a phase space portrait. Indeed SIM is based on the local estimate of the correlation integral for each vector in the phase space, by measuring the "crowding" of the vectors around each individual point in the phase space.

For example, suppose we choose to define vectors of dimension $D$ from a time series $x\left(t_{i}\right)$, with time delay $\Delta t^{\prime}=\Delta t$. If the time series has $N$ observed points, the number of vectors is $N^{\prime}=N-D+1$. Then, for each of the $N^{\prime}$ vectors, say the $i$ th, we calculate the cumulative number function,

$C_{i}(R)=n\left\{j \mid d_{i j} \leq R\right\}$.

In other words, $C_{i}(R)$ measures the number of vectors $j$ whose distance $d_{i j}$ from the vector $i$ is smaller than $R$. To estimate the local scaling properties of the distribution of vectors, one approximates the function $C_{i}(R)$ with a power law of the 
form $C_{i}(R) \sim R^{\alpha_{i}}$ within a given interval $\left[R_{1}, R_{2}\right]$ (which is related to the typical distances between the data points, that, in turn, depend on the choice of the embedding space dimension). The exponent $\alpha_{i}$ is called scaling index. In practice, the scaling index for each vector $i$ is computed by considering the logarithmic increase of $C_{i}(R)$ within a suitably chosen interval $\left[R_{1}, R_{2}\right]$. In all our calculations $\alpha_{i}$ has been determined as follows:

$\alpha_{i}=\frac{\log C_{i}\left(R_{1}, R_{2}\right)-\log C_{i}\left(R_{1}\right)}{\left(\log R_{2}-\log R_{1}\right)}$,

where $C_{i}\left(R_{1}, R_{2}\right)$ denotes the mean cumulative number within the interval $\left[R_{1}, R_{2}\right]$. Note that, by definition, the scaling index cannot have negative values.

The scaling index can be estimated for all the $N^{\prime}$ vectors in the phase space, producing a distribution of scaling indices, whose shape, width and centroid quantify the topological properties of the phase space portrait. In this way, the temporal properties of the original light curve are translated into the properties of the scaling index distribution.

Note that for a purely random process (e.g. a process with an associated time series where the points vary independently) the shape of the histogram is broad, dominated by the rightward tail, and the average scaling index $\langle\alpha\rangle$ tends to the value of the dimension of the embedding space. On the other hand, for highly correlated processes and for deterministic (chaotic) processes, the histogram is typically narrowly shaped, with the value of $\langle\alpha\rangle$ always significantly smaller than the dimension of the embedding space.

One of the most appealing characteristics of SIM is its ability to discern underlying structures in noisy data. Therefore, it might be more sensitive than linear methods like PSD, SF and PDF in identifying intrinsic differences in the temporal properties of 3C 390.3 during 1999 and 2000.

To test the possible presence of two distinct temporal states of the system in the 1999 and 2000 observations, we have estimated the distribution of scaling indices of these two light curves. At this point, we are not interested in determining any kind of "dimension" of the system, since this would be meaningless for non-deterministic systems. Our aim is to compare the 1999 and 2000 histograms of the scaling index values, and try to detect any significant differences between them. To the extent that these histograms correspond to the temporal properties of the system, any differences will imply variations in the intrinsic temporal properties of the system.

We calculated the scaling index for all the vectors of the two phase space portraits derived from the two monitoring campaigns, using embedding spaces of dimensions ranging between $D=2$ and 10 . There are no systematic prescriptions for the choice of the embedding dimension. The discriminating power of the statistic based on the scaling index is enhanced using high embedding dimensions. This can be understood in the following way: if the data are embedded in a lowdimension phase-space many of them will fall on the same position, losing part of the information. On the other hand, the choice of too high embedding dimensions would reduce the point density in the pseudo phase-space, lowering the statistical significance of the test. For the time delay $\Delta t^{\prime}$, we have used $\Delta t^{\prime}=32 \mathrm{~s}$ and $160 \mathrm{~s}$, producing, with the former choice, $\sim 4500-5000$ vectors, and, with $\Delta t^{\prime}=160 \mathrm{~s}$, 1000-1200 vectors, depending on the dimension and on the campaign (the 2000 campaign, albeit shorter, has longer individual observations, producing a slightly higher number of data points and thus of vectors). The use of small time bins causes the data points to be correlated, but this is not necessarily a drawback, in particular in the case where one wants to measure a difference in the degree of correlation of two timeseries. In all the cases (i.e. for all $D$ values) we found that the 1999 scaling index histogram is systematically different from the 2000 histogram, with the former being significantly narrower, and its centroid being smaller than the latter. This result suggests that the points in the 1999 light curve are more "strongly" correlated (e.g., over longer time-scales) than those in the 2000 light curve.

Importantly, the results obtained using tbin $=32 \mathrm{~s}$ are fully consistent with those obtained with tbin $=160 \mathrm{~s}$, suggesting that the scaling index method is actually able to detect the same underlying structure despite the different levels of Poisson noise (by using tbin $=32 \mathrm{~s}$, the ratio between the mean error and the mean count rate $\langle e r r\rangle /\langle$ rate $\rangle$ increases by a factor larger than $80 \%$ compared to tbin $=160 \mathrm{~s})$. The resulting histograms for a 5-dimensional phase space for tbin $=32 \mathrm{~s}$ (top panel) and tbin $=160 \mathrm{~s}$ (bottom panel) are plotted in Fig. 7: the histograms with solid lines represent data from the first monitoring campaign, whereas the histograms with dashed lines refer to 2000. In both panels, the thick solid (dashed) line represents the location of the centroid of $\langle\alpha\rangle$ in 1999 (2000), which remains roughly at the same location despite the large change in the signal-to-noise level.

\subsubsection{Impact of red and Poisson-noise effects on the SIM}

In the previous sections we discussed how the red-noise effects might affect the results from the SF and PDF analyses, casting doubt on the statistical significance of differences observed in the temporal properties between 1999 and 2000. Therefore, it is imperative to assess the impact of the red-noise effects on SIM.

In May 2000 (i.e., near the beginning of the second monitoring campaign of 3C 390.3) the propane layer on PCU0 was damaged, causing a systematic increase of the background. This effect is accounted for by the most recent PCA background models and does not represent a problem when sufficiently large time bins are used (http://lheawww.gsfc.nasa.gov/users/craigm/ pca-bkg/bkg-users.html). However, the SIM, being a statistical method, needs large numbers of points to work properly and thus necessarily requires the use of small time bins. We therefore paid particular attention to possible systematic effects caused by the use of PCU0 data in 2000. Indeed, the SIM does reveal a difference between the PCU0 and PCU2 during 2000, with the former PCU being characterized by larger values of $\langle\alpha\rangle$. We report the SIM results obtained excluding the data from PCU0 in 2000. However, for completeness, we also 

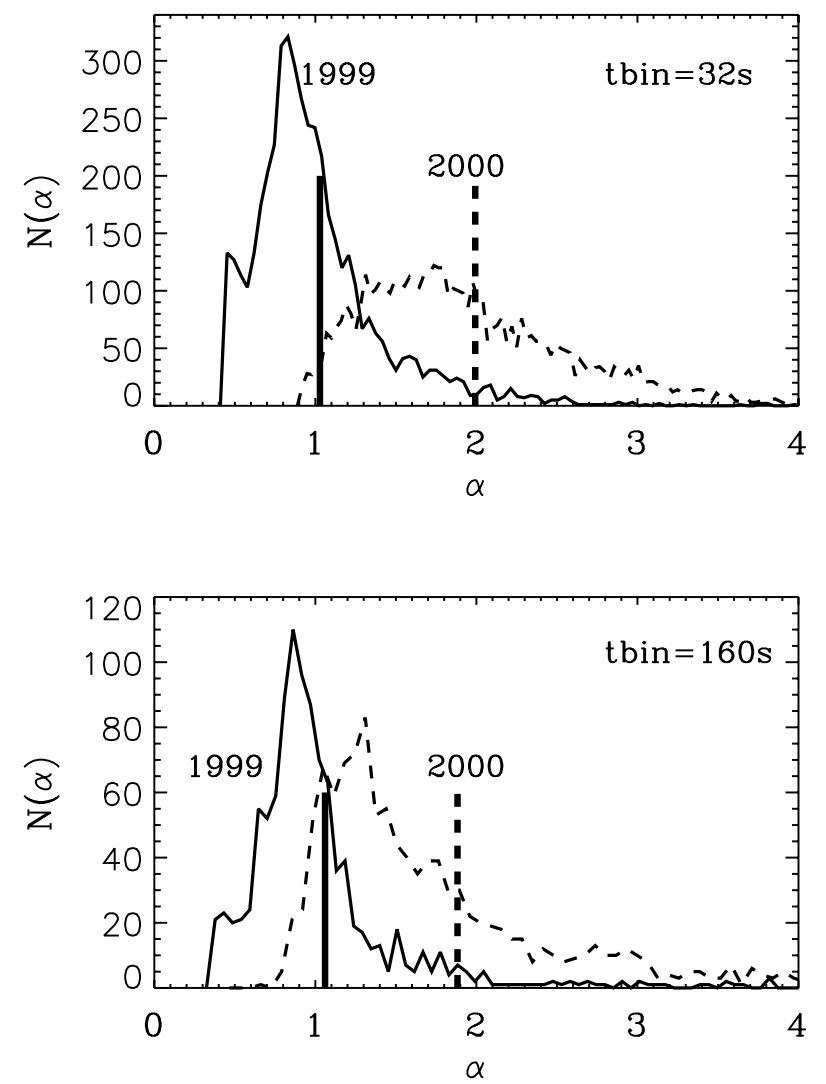

Fig. 7. Scaling index distributions of 3C 390.3 during the 1999 (solid line) and 2000 (dashed line) monitoring campaign, using light curves binned at $32 \mathrm{~s}$ (top panel), and $160 \mathrm{~s}$ (bottom panel). The thick continuous lines represent the scaling index mean value during 1999, $\left\langle\alpha_{99}\right\rangle$, while the thick dashed lines represent $\left\langle\alpha_{00}\right\rangle$.

carried out all tests using both PCUs in 2000, and reached the same conclusions.

To assess the impact of red-noise effects on SIM, we have used $10^{3}$ synthetic light curves obtained from Monte Carlo simulations very similar to those described in Sect. 4.2, the only difference being the use of one PCU (PCU2) instead of two (PCU0+PCU2) for the simulated data of 2000. As before, we report our results in the case when the PSD break at $1 / 300$ day $^{-1}$.

For each pair of synthetic light curves the SIM is applied and the difference $\Delta \alpha \equiv\left\langle\alpha_{00}\right\rangle-\left\langle\alpha_{99}\right\rangle$ is computed. For simplicity and computational reasons (since we are dealing with thousands of simulations of data-sets containing thousands of points), we limit our discussion mainly to $\Delta \alpha$ and $\Delta \sigma_{\alpha}$ (i.e., the difference between the dispersion of the $\alpha$ values around $\langle\alpha\rangle$ ) as the discriminating parameters between the scaling index histograms of the two light curves. However, SIM provides more information, and one can use the full distribution of the scaling index values for the most accurate comparison between the two observed histograms. Figure 8 (left panels) reveals that rednoise effects are unable to explain $\Delta \alpha_{\text {obs }}$ (top panel; thick solid line), $\Delta \sigma_{\text {obs }}$ (bottom panel) at a very high significance level $(P<0.1 \%)$. If no Poisson noise was added to the synthetic light curves, then the distributions of $\Delta \alpha$ and $\Delta \sigma$ would be centered around zero. The fact that the distributions are shifted towards positive values is due to the use of two PCUs in 1999 and only one in 2000, and hence to the different Poisson noise contributions to the 1999 and 2000 synthetic data.

To understand these results better, we have also plotted the distributions of the scaling index centroids and standard deviations in 1999 and 2000 (Fig. 8 middle and right panels, respectively). It is clearly shown that the distributions of simulated data of 1999 (Fig. 8 middle panels) are marginally consistent with the corresponding observed values: the chance probabilities to obtain values consistent with $\left\langle\alpha_{99}\right\rangle$ and $\sigma_{\alpha, 99}$ are $P \sim 6 \%$ and $P \sim 15 \%$, respectively. In contrast, the distributions of simulated data of 2000 (Fig. 8 right panels) are inconsistent with the observed values $\left(P<0.1 \%\right.$ for both $\left\langle\alpha_{00}\right\rangle$ and $\sigma_{\alpha, 00}$ distributions).

The fact that $\Delta \alpha_{\text {synth }}$ and $\Delta \sigma_{\text {synth }}$ are not centered around zero, because of the presence of different Poisson noise components in the two light curves, motivated us to further investigate the impact of Poisson noise on the distribution of the scaling index values. In principle, the effects of Poisson noise have been already accounted for by the red-noise simulations, where the "appropriate" noise level has been computed also considering the different background levels during 1999 and 2000. Nevertheless, we considered what the effects on the SIM results would be if we add, arbitrarily, some artificial extra Poisson noise (equal to half of the noise actually present in the data) to the synthetic light curves. Thus, we consider the possibility that the "real" instrumental noise in the light curves is, for some unknown reason, underestimated in the previous simulations, and what effects this may have on the SIM results.

The results of the noisy simulations are plotted in Fig. 9. Based on the $\Delta \alpha_{\text {synth }}$ distribution only (top left panel), we would conclude that the simulated results are in agreement with the observed value of $\Delta \alpha$ (the chance probability is indeed $P \sim 17 \%$ ). This might lead to the erroneous conclusion that the differences observed using SIM could be simply ascribed to the fact that the Poisson noise in the previous simulations was not accounted for correctly. However, the $\Delta \sigma_{\text {synth }}$ distribution (Fig. 9, bottom left panel; $P<0.1 \%$ ) reveals that this is not the case. The same conclusion is reached with the synthetic distributions in 1999 and 2000, and keeping in mind that, in order to infer consistency between simulated and observed values, the distributions of both scaling index centroids and standard deviations should be consistent with the respective observed values. This is clearly not the case in 1999 and in 2000: in the first case, the synthetic distribution of $\left\langle\alpha_{99}\right\rangle$ is very poorly consistent with the observed value $(P=0.1 \%)$, whereas in 2000 , it is the synthetic distribution of $\sigma_{\alpha_{00}}$ values that is inconsistent with $\sigma_{\alpha_{00, \mathrm{obs}}}(P<0.1 \%)$.

Another macroscopic consequence of the arbitrary increase of the Poisson noise is the substantial right-ward shift of $\langle\alpha\rangle$. This might appear at odds with the lack of shift in $\langle\alpha\rangle$ observed in the real data when passing from tbin $=160 \mathrm{~s}$ to tbin $=32 \mathrm{~s}$ (see Fig. 7). However, this apparent discrepancy can be explained by the most important property of the SIM, i.e. its ability to discern an underlying structure in noisy data, if an underlying structure is present. On the other hand, if no underlying structure is present (as in the case of the simulated 

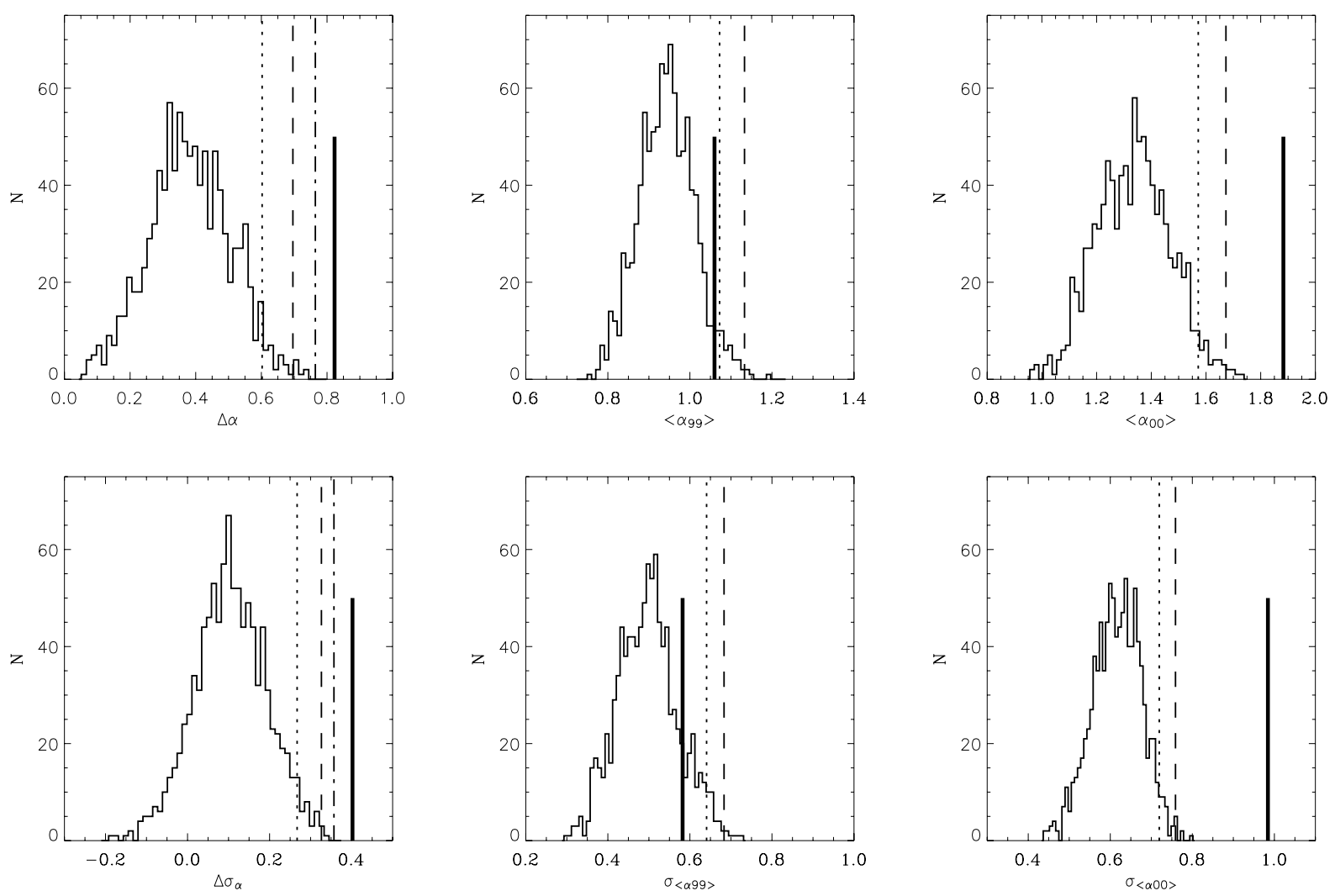

Fig. 8. Top left panel: distribution of $\Delta \alpha_{\text {synth }}$ obtained from red-noise simulations (tbin $=160 \mathrm{~s}$ ) compared to $\Delta \alpha_{\text {obs }}$ (thick solid line). The dotted, dashed and dot-dashed lines represent the 95\%, 99\%, and 99.9\% confidence levels, respectively. Top middle and right panels: distribution of mean synthetic scaling indices obtained from red-noise simulations, $\left\langle\alpha_{99(00) \text {,synth }}\right\rangle$, compared with $\left\langle\alpha_{99(00), \text { obs }}\right\rangle$ (thick solid line). Bottom left panel: distribution of $\Delta \sigma_{\text {synth }}$, i.e., of the differences between standard deviations around $\langle\alpha\rangle$ of the synthetic distributions of scaling indices. Bottom middle and right panels: distribution of synthetic standard deviations around $\langle\alpha\rangle$ in 1999 and 2000, respectively.

red-noise data), the increase of noise (and hence of randomness) simply causes a shift of $\langle\alpha\rangle$ to larger values.

An alternative and model-independent way to assess if $\Delta \alpha_{\mathrm{obs}}$ is dominated by Poisson noise effects is based on the shuffling of the temporal order of the RXTE orbits. In this way, the original long-term structure is destroyed, while the Poisson noise levels and all the statistical moments of the distributions in 1999 and 2000 are unaffected. If the orbit-shuffled light curves yield a $\Delta \alpha$ consistent with $\Delta \alpha_{\text {obs }}$, then we should conclude that the difference in Poisson noise between the 1999 and 2000 (along with the difference in the short-term intra-orbit variability) is the simplest explanation of the difference in scaling index observed. On the other hand, if the values of $\Delta \alpha_{\text {shuffled }}$ are not consistent with the observed value, the direct consequence is that the long-term temporal structure of the two original light curves does play an important role.

The results of this test, carried out using light curves with tbin $=32 \mathrm{~s}$ because of their higher Poisson noise level (but similar results are obtained with tbin $=160 \mathrm{~s}$ ), are shown in Fig. 10. The left panel shows that the orbit-shuffled results are inconsistent with the $\Delta \alpha_{\text {obs }}$ at very high significance level. The middle and right panels show the distributions of the centroid $\langle\alpha\rangle$ and the width $\sigma_{\alpha}$ of the scaling index histograms obtained by shuffling the orbit order in 1999 (very similar results are obtained with 2000 data). Both plots show that the orbit-shuffled quantities tightly cluster at a location that is, statistically speaking, far away from the real data, indicating that the long-term temporal structure contributes substantially to the SIM results.

We conclude that, contrary to the results based on the comparison between the 1999 and 2000 SFs and PDFs, the observed differences between the respective distributions of the scaling index values are almost certainly intrinsic, i.e. they cannot be explained by Poisson noise, red-noise, or other instrumental effects. To the extent that the differences of the scaling index histograms correspond to differences in the temporal properties of the two light curves, we consider them as significant evidence of non-stationarity in the the X-ray emitting process in 3C 390.3 .

\section{Spectral variability}

After searching systematically for evidence of temporal changes in 3C 390.3, we now investigate whether the apparent changes of the statistical timing properties are accompanied by spectral variations, and what the nature of the spectral changes is (e.g., is 3C 390.3 experiencing an actual state transition?). To this end, we have performed two analyses.

1. The first (model-independent) method is based on the X-ray color - count rate plot, often used for GBHs to separate the spectral states. The plot of X-ray color (i.e., the ratio 

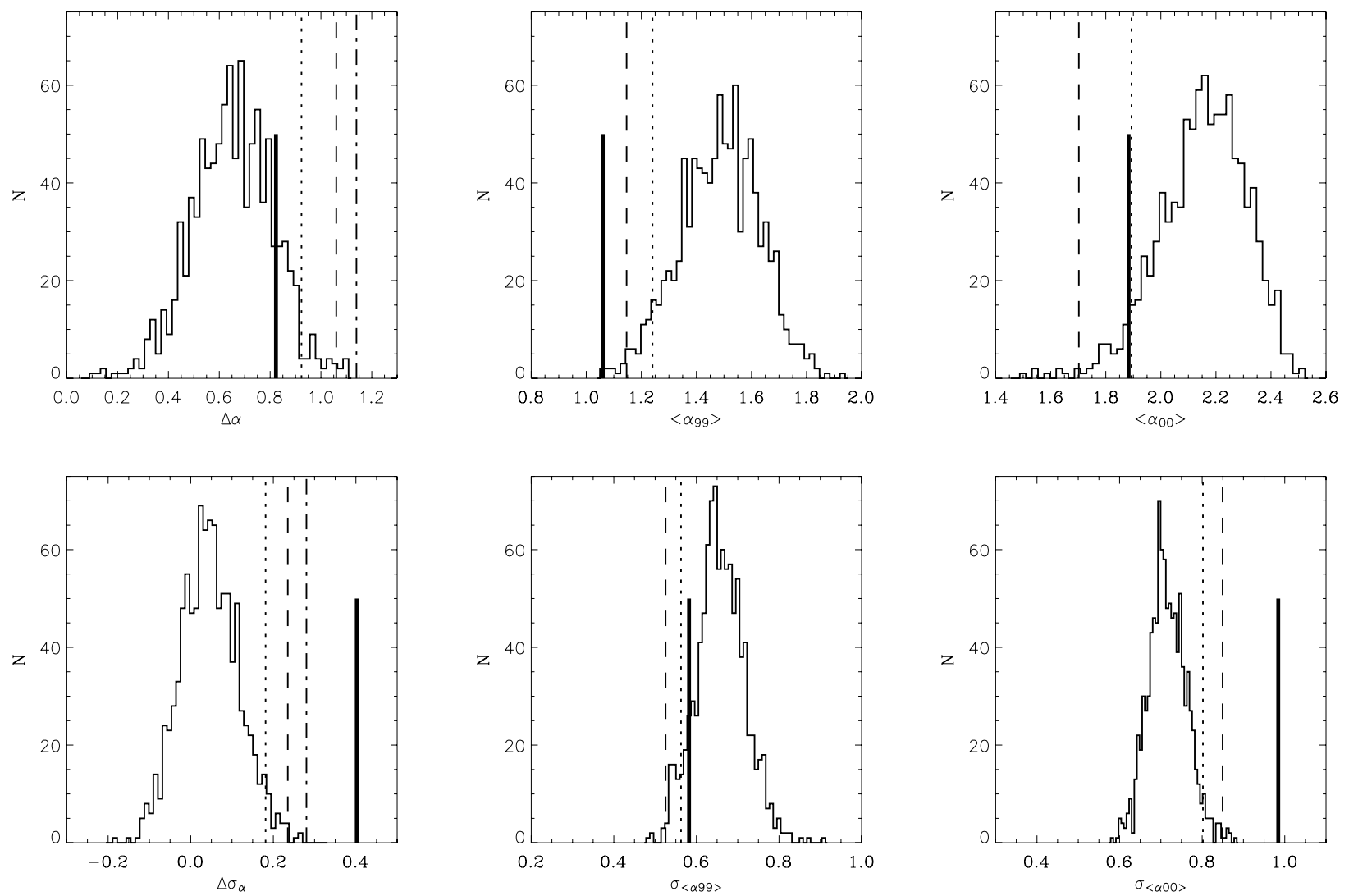

Fig. 9. Top left panel: distribution of $\Delta \alpha_{\text {synth }}$ obtained from red-noise simulations with $50 \%$ extra noise compared with $\Delta \alpha_{\text {obs }}$ indicated by the thick continuous line (tbin $=160 \mathrm{~s}$ ). Top middle and right panels: distribution of mean synthetic scaling indices obtained from red-noise simulations with $50 \%$ extra noise, $\left\langle\alpha_{99(00), \text { synth }}\right\rangle$, compared with $\left\langle\alpha_{99(00), \text { obs }}\right\rangle$ (thick continuous line). The dotted and dashed lines represent the 95\% and 99\% confidence levels, respectively. Bottom left panel: distribution of $\Delta \sigma_{\text {synth }}$, i.e., of the differences between standard deviations around $\langle\alpha\rangle$ of the synthetic distributions (with 50\% extra noise) of scaling indices. Bottom middle and right panels: distribution of synthetic standard deviations around $\langle\alpha\rangle$ in 1999 and 2000, respectively.
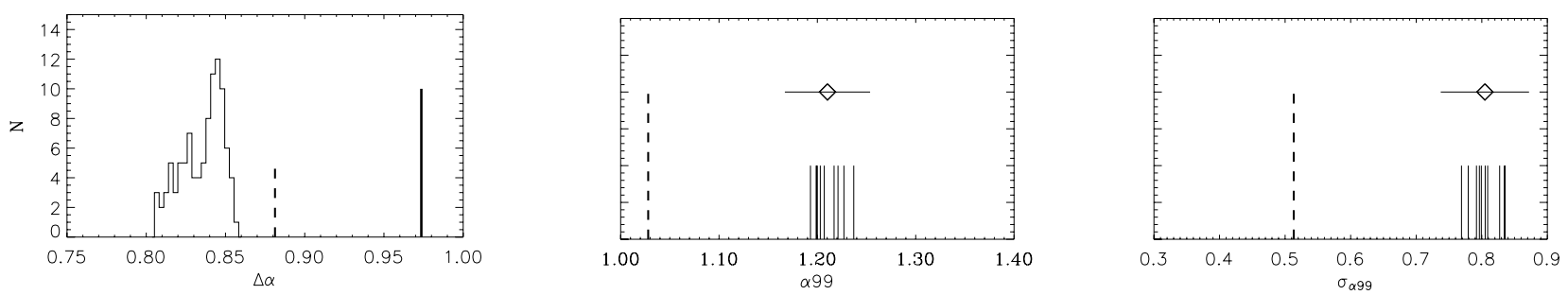

Fig. 10. Left panel: distribution of $\Delta \alpha$ obtained by shuffling the time order of the RXTE orbits. The thick solid line is the location of $\Delta \alpha_{\mathrm{obs}}$. Middle panel: distribution of $\langle\alpha\rangle$ (short, solid lines) obtained by shuffling the orbits order in 1999 (tbin $=32 \mathrm{~s}$ ). The open diamond gives the mean value of the shuffled data and its error bar represents the $3 \sigma$ confidence level. The long, dashed line represents the original value, obtained without any shuffling. The same symbol convention is used for the right panel, showing the distribution of $\sigma_{\alpha}$ in 1999.

of the 7-20 over the $2-5 \mathrm{keV}$ count rate) versus the total 2-20 keV count rate is shown in Fig. 11. The hardness ratio clearly decreases with increasing flux, during the second part of the 1999 and the whole of the 2000 campaigns. This trend is typical of type 1 radio-quiet AGN, and has been observed many times (e.g., Papadakis et al. 2002; Taylor et al. 2004, and references therein). This supports the conclusion of Gliozzi et al. (2002) that the X-ray emission in 3C 390.3 is not jet-dominated.

During the first half of the 1999 campaign, the source was at its lowest flux state. The spectrum of the source also appears to be quite hard, consistent with the general trend of spectral hardening towards low flux states. However, the scatter of the hardness ratio points is very large, and it seems that during this state, the spectral variations are independent of the source flux variations. So, based on the color-count rate plot, it seems possible that during this period the X-ray emission mechanism may behave in a different way, although the errors associated with the X-ray colors (see Fig. 1 bottom panel) prevent us from reaching a firm conclusion. 


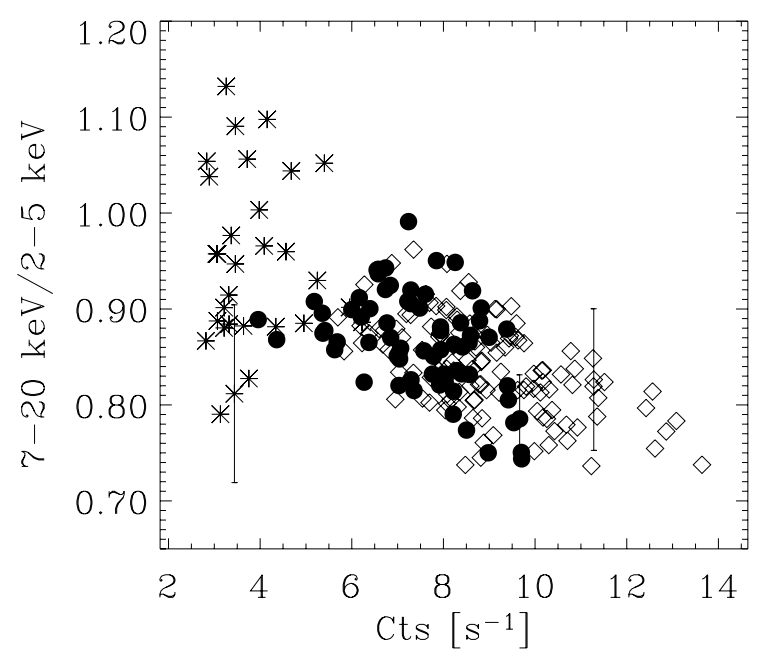

Fig. 11. 7-20 keV/2-5 keV X-ray color plotted versus the total count rate of 3C 390.3. Star symbols correspond to the first 200 days of the 1999 light curve, the filled dots describe the second part of the 1999 campaign, and the open diamond the 2000 light curve. For the sake of clarity, only a characteristic error for each cluster of points has been plotted. All the errors associated with the X-ray colors are reported in Fig. 1 bottom panel. Time bins are $5760 \mathrm{~s}$ ( $\sim 1$ RXTE orbit).

Table 1. Time intervals used for time-resolved spectral analysis.

\begin{tabular}{ccccc}
\hline \hline $\begin{array}{c}\text { Start time } \\
(\mathrm{y} / \mathrm{m} / \mathrm{d} \mathrm{h}: \mathrm{m})\end{array}$ & $\begin{array}{c}\text { End time } \\
(\mathrm{y} / \mathrm{m} / \mathrm{d} \mathrm{h}: \mathrm{m})\end{array}$ & $\begin{array}{c}\text { Exposure } \\
(\mathrm{ks})\end{array}$ & Flux $^{a}$ & $\Gamma$ \\
\hline 99/01/08 00:26 & $99 / 02 / 2517: 58$ & 17.90 & 1.87 & $1.55_{0.05}^{0.08}$ \\
99/02/28 16:48 & $99 / 05 / 1101: 05$ & 24.10 & 1.68 & $1.54_{0.05}^{0.03}$ \\
99/05/14 00:58 & $99 / 07 / 1020: 15$ & 21.20 & 2.64 & $1.64_{0.04}^{0.03}$ \\
99/07/13 08:56 & $99 / 09 / 1116: 03$ & 22.56 & 3.86 & $1.68_{0.03}^{0.02}$ \\
$99 / 09 / 1418: 07$ & $99 / 11 / 1322: 58$ & 23.38 & 3.71 & $1.67_{0.02}^{0.02}$ \\
$99 / 11 / 1714: 25$ & $00 / 01 / 0308: 00$ & 18.69 & 4.71 & $1.74_{0.02}^{0.02}$ \\
$00 / 01 / 0611: 50$ & $00 / 02 / 2906: 57$ & 15.26 & 3.84 & $1.66_{0.03}^{0.02}$ \\
$00 / 03 / 0304: 26$ & $00 / 05 / 1112: 43$ & 32.46 & 4.90 & $1.70_{0.02}^{0.01}$ \\
$00 / 05 / 1408: 41$ & $00 / 07 / 0401: 22$ & 22.50 & 3.80 & $1.61_{0.02}^{0.03}$ \\
$00 / 07 / 0700: 09$ & $00 / 09 / 0122: 29$ & 26.91 & 4.10 & $1.63_{0.02}^{0.02}$ \\
$00 / 09 / 055: 52$ & $00 / 11 / 1012: 12$ & 32.22 & 3.91 & $1.62_{0.02}^{0.01}$ \\
$00 / 11 / 1312: 57$ & $00 / 12 / 3103: 12$ & 25.47 & 5.03 & $1.67_{0.02}^{0.02}$ \\
$01 / 01 / 0308: 53$ & $01 / 02 / 2400: 26$ & 24.45 & 3.46 & $1.61_{0.02}^{0.02}$ \\
\hline
\end{tabular}

a $2-10 \mathrm{keV}$ flux in units of $\left(10^{-11} \mathrm{erg} \mathrm{cm}^{-2} \mathrm{~s}^{-1}\right)$, calculated assuming a power law plus Gaussian line model.

2. More stringent constraints can be derived by using a second (model-dependent) method: the time-resolved spectral analysis. Since the data consist of short snapshots spanning a long temporal baseline, they are well suited for monitoring the spectral variability of the source.

We divided the light curve of Fig. 1 into thirteen intervals, of duration 50-60 days each, with net exposure times between $15 \mathrm{ks}$ and $32 \mathrm{ks}$. This scheme represents a trade-off between the necessity to isolate intervals with different but well defined average count rates and the need to reach a signalto-noise ratio $(\mathrm{S} / \mathrm{N})$ high enough for a meaningful spectral

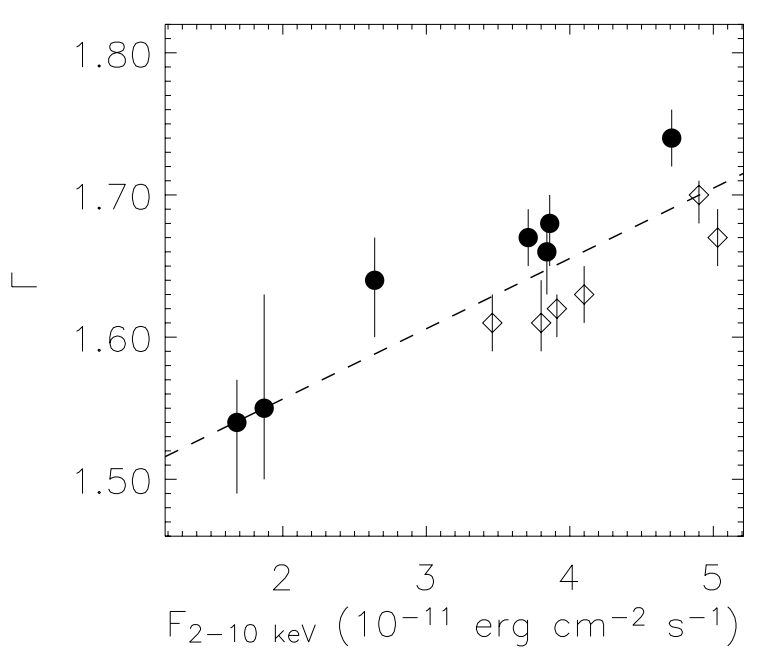

Fig. 12. Photon index, $\Gamma$, plotted versus the $2-10 \mathrm{keV}$ flux $\left(10^{-11} \mathrm{erg} \mathrm{cm}^{-2} \mathrm{~s}^{-1}\right)$. Filled dots refers to the 1999 campaign, and the open diamond to the 2000 light curve. The dashed line represents a linear least squares fit to all the data points.

analysis. The dates, exposure times and mean fluxes in the thirteen selected intervals are listed in Table 1.

We fitted the 4-20 keV spectra with a model consisting of a power-law, absorbed by the Galactic absorbing column, plus a Gaussian $\mathrm{Fe} \mathrm{K} \alpha$ line with a rest energy fixed at $6.4 \mathrm{keV}$. In all the intervals, the line is statistically required at more than $99 \%$ confidence level, according to an F-test. However, due to the low $\mathrm{S} / \mathrm{N}$, the line parameters are characterized by large errors ( $\sim 100 \%$ on the line flux and width) and thus their evolution in time cannot be investigated properly. On the other hand, the photon indices are well constrained with uncertainties of the order of $1-3 \%$. Figure 12 shows the plot of the photon index $\Gamma$ versus the $2-10 \mathrm{keV}$ flux, with the filled dots representing intervals during the 1999 campaign and open diamonds during the 2000 campaign. The photon index increases steadily with the $2-10 \mathrm{keV}$ flux, without any indication of a discontinuity between the $\Gamma$ corresponding to the low state and the other photon indices.

Based on this analysis, we conclude that 3C 390.3 is not undergoing a transition between two distinct spectral states during the RXTE ' 99 and ' 00 monitoring campaigns.

\section{Discussion}

We have studied two one-year long, well sampled X-ray light curves of 3C 390.3 from the RXTE archive, in search of evidence for variations in its temporal properties. Our motivation stems from the fact that a long-term ROSAT light curve of the this broad-line radio galaxy had shown evidence of nonlinearity and possibly non-stationarity in the past (Leighly \& O'Brien 1997). The light curves we have used are the longest and best sampled light curves on time-scales of days/months of this source to date. Hence, they offer a good opportunity to investigate the important issue of stationarity in the X-ray light curves of AGN. 


\subsection{Multi-technique timing approach: linear methods}

In order to assess whether the light curve of 3C 390.3 shows variations in its temporal properties, we performed a thorough temporal analysis based on different complementary timing techniques and extensive Monte Carlo simulations. The first advantage of this approach is that it leads to more robust results, not biased by the specific characteristics of a single method. In addition, since the diverse methods employed probe different statistical properties, they provide us with complementary information that can be combined to constrain the physical mechanism underlying the observed variability.

The power spectral density analysis results suggest the possible shift of the frequency break between 1999 and 2000, with $t_{\text {break }}$ moving from $\sim 1 / 300-1 / 400$ days to $\sim 1 / 50-1 / 100$ days. However, this apparent shift is not of high statistical significance. The structure function analysis also indicates a similar change in the characteristic time-scale of the system. Both SFs have a power-law shape up to lags shorter than $\sim 60$ days. At longer time-scales, the $2000 \mathrm{SF}$ appears to flatten to a roughly constant level, while the 1999 SF keeps rising to the largest time lags sampled. Finally, the comparison between the two probability density Functions (see Fig. 5), suggest the existence of an intrinsic difference between the two time series, with the 1999 data characterized by a bimodal distribution and the 2000 data following a uniform distribution.

However, based on the results from Monte Carlo red-noise simulations, which were employed to test the significance of the apparent differences between the 1999 and 2000 SF and PDFs, we found that they can be the result of the red-noise character of the variability process with a probability at least as high as $\sim 10 \%$.

\subsection{The scaling index method}

In contrast with the linear methods, that showed only suggestive evidence of a change in the temporal properties of 3C 390.3 but were unable to rule out a major influence of red-noise effects, the scaling index method clearly reveals the presence of intrinsic changes that cannot be explained by red-noise or Poisson effects $(P<0.1 \%)$.

It is difficult to understand the consequences of this result. One possibility is that the variability process of 3C 390.3 holds more information than the red-noise PSD reveals. The main property of the synthetic light curves that we have produced using the method of Timmer \& Koening (1995) is that their PSD has a specific red-noise shape. The disagreement between the results from the simulated light curves and the observed SIM values could imply that there is "something" more than just "red plus Poisson noise" in the observed light curves. In fact, there have been indications that this may be the case. For example, Uttley et al. (2004) have shown that the rmsflux relation recently discovered in the X-ray light curves of AGN and GBHs implies that their light curves are intrinsically "non-linear". The simulations that we have used in this work to test the SIM results do not reproduce this rms-flux relation. Perhaps then, the X-ray light curve of 3C 390.3 is non-linear as well, and the differences that we detect with the SIM method reflect this property. Advanced simulations (which are beyond the scope of the present work) are needed to investigate this issue further, and examine whether non-linear synthetic light curves which do reproduce the rms-flux relation are consistent with our results.

On the other hand, it is also possible that, irrespective of whether the light curves are linear or non-linear, the SIM differences that we observe imply real differences in the temporal properties of the source between 1999 and 2000. We should try to identify these properties, in order to advance our understanding of the variability mechanism. The nature of the AGN time variability is still poorly known and apart from the "linear vs. non-linear" issue, one has to consider other issues such as "stochastic vs. chaotic". At the moment, no clear physical insights can be derived from this non-linear tool. Indeed, as pointed out by Vio et al. (1992), a naive application and interpretation of the non-linear method results may lead to conceptual errors.

Nevertheless, the much narrower 1999 scaling index distribution, with its centroid $\langle\alpha\rangle$ located at a value significantly lower than $\left\langle\alpha_{00}\right\rangle$, is consistent with the assumption that the 1999 time series is characterized by a "higher degree of correlation". Since the correlation structure of a time series is characterized by its auto-covariance function, this result may suggest that the the width of the auto-covariance function has decreased during the 2000 observations. This is qualitatively in agreement with the hypothesis that the characteristic time-scale during 1999 is longer than during 2000, as suggested by the PSD and SF analyses. However alternative hypotheses cannot be ruled out yet. If true, the differences in the scaling indices do not challenge the "red plus Poisson noise" view; they simply demonstrate that the first term in this expression may vary with time.

\section{3. $3 C$ 390.3 and the analogy between AGN and $\mathrm{GBHs}$}

It has been suggested and is now widely believed, that AGN are simply the high mass analogs of GBHs. For example, PSD analyses of long, high signal-to-noise, well sampled X-ray light curves of a few AGN have shown clearly that their power spectra are very similar to those of GBHs (e.g. Uttley et al. 2002; Markowitz et al. 2003; McHardy et al. 2004).

One of the most interesting aspects of the X-ray variability behavior of GBHs is that they often show transitions to different "states". "State" transitions have not been observed so far in AGN. The energy spectral results reported in Sect. 5 clearly argue against the presence of a bona fide state transition in 3C 390.3 as well. This is not unexpected if we consider the time-scales at play: in GBH state transitions take place on time-scales of days (e.g. Zdziarski et al. 2004) which translate into thousands of years for 3C 390.3, assuming a linear scaling between the black hole masses.

However, even within the same state, the timing properties of GBHs, like the PSD characteristic time-scales, vary with time. For example, during the "low/hard state", the characteristic time-scales in the X-ray power spectrum of Cyg X-1 change 
with time in a correlated way with the spectral variations of the source (Belloni \& Hasinger 1990; Pottschmidt et al. 2003). This is one example of "stationarity-loss", which can offer important clues on the mechanism responsible for the observed variations. These variations of the temporal properties can take place on time-scales as short as tens of seconds (e.g. Casella et al. 2004). These time-scales are consistent with the length of the 1999 and 2000 observations of 3C 390.3 which we study in this work, assuming again that characteristic time-scales scale linearly with black hole mass.

We find that the energy spectrum of the source in the first part of the 1999 light curve is significantly harder than that of the high flux peaks after day 400 (Fig. 1). Furthermore, the SIM results show variations in the timing properties of the source during the same periods. In itself, this result strengthens the analogy between the X-ray variability properties of AGN and GBHs: as in the Galactic systems, 3C 390.3 shows simultaneous spectral and intrinsic timing property variations.

However, as we discussed above, the SIM results are not easy to interpret at the moment. On the other hand, the results presented in Sects. 4.1-4.3 do suggest a much closer analogy between 3C 390.3 and Cyg X-1, but this is not statistically significant, possibly because the quality of the light curves at hand is not good enough.

Our results show that the spectral slope, $\Gamma$, was $\sim 1.55$ during the first $\sim 150$ days of the 1999 monitoring observations, increasing by a factor of $\sim 10 \%$ during the peak flux periods of the 2000 monitoring observations. The Cyg X-1 slope does not become as hard as 1.55 , nevertheless, according to the results of Pottschmidt et al. (2003), when $\Gamma$ increases from its lowest value of 1.8 to a value of $\sim 1.95-2$ (an increase by a factor of $\sim 10 \%$ as well) the centroid frequency of the $L_{2}$ component in its power spectrum increases by a factor of $\sim 5-6$ (as shown in the middle panel of their Fig. 7). Since the $L_{2}$ centroid frequency is commonly thought to correspond to the "highfrequency" PSD breaks in AGN, this is the same behavior that the results of 3C 390.3 suggest.

As mentioned above, we believe that the temporal and spectral behavior of 3C 390.3, as seen by RXTE during the 1999 and 2000 monitoring campaigns, qualitatively mimics the properties of the GBH Cyg X-1 during the low/hard state, provided that the appropriate time-scale differences are taken into account.

\section{Summary and conclusions}

The main results and conclusions of this work can be summarized as follows:

- We have studied in detail the variability characteristics of the two-year long, RXTE monitoring light curve of 3C 390.3. Linear methods, like PSD, SF and PDF analysis show suggestive evidence of a change in the characteristic time-scale of the system between 1999 and 2000.

- However, only the results from the scaling index method show clear and significant evidence of a change in the intrinsic properties of the system that cannot be explained by red or Poisson noise effects.
- It is not easy to interpret the SIM results at this moment. To the extent that the scaling index is related to the timing properties of the variability process, one possibility is a change of the characteristic time-scale between 1999 and 2000, in agreement with the suggestive results from the PSD, SF and PDF analyses.

- Our results reinforce the similarity between the X-ray variability properties of AGN and GBHs. In analogy with GBH phenomenology, the loss of stationarity in 3C 390.3 seems to correspond to an increase in the frequency break as the spectrum softens, as observed in Cyg X-1, when the system is in its low/hard spectral state.

- Since the SIM is not yet a standard technique, further work is needed to investigate its properties and its possible relation to more frequently used (and better understood) statistical analysis tools like the power spectrum. Nevertheless, we believe that the present results demonstrate the benefits of the application of the SIM (and possibly other non-linear methods) in the analysis of AGN X-ray light curves.

- The RXTE archive contains numerous, long AGN light curves appropriate for a systematic study to search for spectrally related variations of the timing properties of these systems. Since the outcome of any Monte Carlo simulation based on an assumed "model-PSD" is inevitably dependent on the assumptions made, a thorough analysis of a large number of high-quality AGN light curves is likely the most direct way to examine whether the present results are indicative of AGN behavior in general. We plan to explore this issue in a future work.

Acknowledgements. We thank the anonymous referee for the useful comments and suggestions. We are grateful to H. Scheingraber for providing the SIM code. We thank P. Uttley, S. Vaughan and A. Markowitz for the lengthy discussions and their criticisms of the original draft of this paper, and the methodology used herein. Financial support from NASA LTSA grants NAG5-10708 (MG) is gratefully acknowledged.

\section{References}

Arnaud, K. 1996, in Astronomical Data Analysis Software and Systems V, ed. G. Jacoby, \& J. Barnes (San Francisco: ASP), ASP Conf. Ser., 101, 17

Atmanspacher, H., Scheingraber, H., \& Wiedenmann, G. 1989, Phys. Rev. A, 40, 3954

Belloni, T., \& Hasinger, G. 1990, A\&A, 227, L33

Bregman, J. N., Glassgold, A. E., Huggins, P. J., et al. 1990, ApJ, 352, 574

Casella, P., Belloni, T., Homan, J., \& Stella, L. 2004, A\&A, 426, 587

Edelson, R., Alexander, T., Crenshaw, D. M., et al. 1996, ApJ, 470, 364

Gliozzi, M., Brinkmann, W., Räth, C., et al. 2002, A\&A, 391, 875

Gliozzi, M., Sambruna, R., \& Eracleous, M. 2003, ApJ, 584, 176

Grassberger, P., \& Procaccia, I. 1983, Phys. Rev. Lett. A, 51, 346

Green, A. R., McHardy, I. M., \& Lehto, H. J. 1999, MNRAS, 305, 309

Jahoda, K., Swank, J., Giles, A. B., et al. 1996, Proc. SPIE, 2808, 59

Kaspi, S., Smith, P. S., Netzer, H., et al. 2000, ApJ, 533, 631 
Kantz, H., \& Schreiber, T. 1997, Nonlinear Time Series Analysis (Cambridge University Press)

Lehto, H. J., Czerny, B., \& McHardy, I. M. 1993, MNRAS, 261, 125

Leighly, K., \& O’Brien, P. 1997, ApJ, 481, L15

Lyubarsky, Yu. E. 1997, MNRAS, 292, 679 46, 97

Markowitz, A., Edelson, R., Vaughan, S., et al. 2003, ApJ, 593, 96

McClintock, J. E., \& Remillard, R. A. 2003, to appear in Compact Stellar X-ray Sources, ed. W. H. G. Lewin, \& M. van der Klis (Cambridge University Press) [arXiv: astro-ph/0306213]

McHardy, I. M., Papadakis, I. E., Uttley, P., Page, M. J., \& Mason, K. O. 2004, MNRAS, 348, 783

Mineshige, S., Ouchi, B., \& Nishimori, H. 1994, PASJ, 46, 97

Nandra, K., Clavel, J., Edelson, R. A., et al. 1998, ApJ, 505, 594

Papadakis, I. E., \& Lawrence, A. 1993, MNRAS, 261, 612

Papadakis, I. E., \& Lawrence, A. 1995, MNRAS, 272, 161

Papadakis, I. E., Petrucci, P. O., Maraschi, L., et al. 2002, ApJ, 573, 92

Peterson, B. M., Ferrarese, L., Gilbert, K. M., et al. 2004, ApJ, 613, 682
Pottschmidt, K., Wilms, J., Nowak, M. A., et al. 2003, A\&A, 407, 1039

Räth, C., \& Morfill, G. 1997, J. Opt. Soc. Am. A, 14, 3208

Räth, C., Bunk, W., Huber, M. B., et al. 2002, MNRAS, 337, 413

Rotschild, R. E., Blanco, P. R., Gruber, D. E., et al. 1998, ApJ, 496, 538

Sergeev, S., Pronik, V. I., Peterson, B. M., Sergeeva, E. A., \& Zheng, W. 2002, ApJ, 576, 660

Simonetti, J. H., Cordes, M. J., \& Heeschen, D. S. 1985, ApJ, 296, 46 Taylor, R. D., Uttley, P., \& McHardy, I. M. 2004, MNRAS, 342, L31

Terrel, N. J. 1972, ApJ, 174, L35

Timmer, \& Koenig 1995, A\&A, 300, 707

Uttley, P., \& McHardy, I. M. 2001, MNRAS, 323, L26

Uttley, P., McHardy, I. M., \& Papadakis, I. E. 2002, MNRAS, 332, 231

Vaughan, S., Edelson, R., Warwick, R. S., \& Uttley, P. 2003, MNRAS, 345,1271

Vio, R., Cristiani, S., Lessi, O., \& Provenzale, A. 1992, ApJ, 391, 518

Zdziarski, A. A., Gierlinski, M., Mikolajewska, J., et al. 2004, MNRAS, 351, 791 